\title{
The variability and periods in the BL Lac AO $0235+164$
}

\author{
J. H. Fan ${ }^{1,2}$, O. Kurtanidze ${ }^{3}$, X. Liu ${ }^{4}$, Y. Liu ${ }^{1,2}$, H. T. Wang ${ }^{5}$,

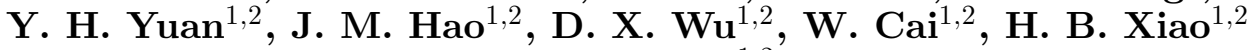 \\ and Z. Y. Pei $\mathbf{i}^{1,2}$ \\ ${ }^{1}$ Center for Astrophysics, Guangzhou University, Guangzhou 510006, China \\ email: fjh@gzhu.edu.cn \\ ${ }^{2}$ Astronomy Science and Technology Research Laboratory of Department of Education of \\ Guangdong Province, Guangzhou 510006, China \\ ${ }^{3}$ Abastumani Observatory, Mt. Kanobili, 0301 Abastumani, Georgia \\ ${ }^{4}$ Xinjiang Astronomical Observatory, Chinese Academy of Sciences, Urumqi 830011, China \\ ${ }^{5}$ Faculty of Physics and Electronic Information, LangFang Teacher's College, China
}

\begin{abstract}
Variability is one of the extreme observational properties of BL Lacertae objects. AO $0235+164$ is a well studied BL Lac through the whole electro-magnetic wavebands, it is violently variable in the optical bands. In the present work, we show its optical $\mathrm{R}$ band photometric observations carried out during the period of Nov. 2006 to Dec. 2012 using the Ap6E CCD camera attached to the primary focus of the $70 \mathrm{~cm}$ meniscus telescope at Abastumani Observatory, Georgia. It shows a large variation of $\Delta R=4.88 \mathrm{mag}$ (14.20 - $19.08 \mathrm{mag}$ ) during our monitoring period. When periodicity analysis methods are adopted to its $\mathrm{R}$ observations from our Abastumani monitoring programme and those in the literature, the signs of some periods, $P_{1}=8.26 \mathrm{yr}, P_{2}=0.55 \mathrm{yr}, P_{3}=0.85 \mathrm{yr}, P_{4}=1.99 \mathrm{yr}$ are found.
\end{abstract}

Keywords. galaxies: BL Lacertae Objects: individual (AO 0235+164): photometry

\section{Introduction}

The BL Lac AO $0235+164$, located at $\mathrm{z}=0.94$ is a well studied object. It is observed from radio to $\mathrm{X}$-rays bands, and even high energy $\gamma$-ray regimes. It shows variability timescale from a few days to several years (Webb et al. 1998, Romero et al. 2000, Chen \& Jiang 2001, Fan et al. 2002, Peng \& de Bruyn 2004, Gupta et al. 2008, Rani et al. 2009, and Wang 2014). Raiteri et al. (2001) analyzed about 25 years of observational data in optical and radio bands during the period from 1975 to 2000, and found a quasiperiodicity of the main radio (and optical) outbursts on 5.7 years time scale. Periodicity analysis is also performed to its light curves by us (Fan et al. 2002; Wang 2014). The long-term quasi-period in the light curve perhaps suggests existence of a binary black hole system at its center (Romero et al. 2003; Ostorero, Villata \& Raiteri 2004). AO $0235+164$ is one of the monitored objects in our monitoring programme at Abastumani Observatory, Georgia (Kurtanidze et al. 2009, Nikolashvili \& Kurtanidze 2007 and Fan et al. 2014).

\section{Observations and results}

Abastumani Observatory is located at the top of Mt. Kanobili in the South-Western part of Georgia. Observations are made using a $70 \mathrm{~cm}$ meniscus telescope (f/3), to which a Peltier cooled ST-6 CCD imaging camera was attached to the Newtonian focus from March 1997 to Sept 2006. All our observations are made using the filters combined of 


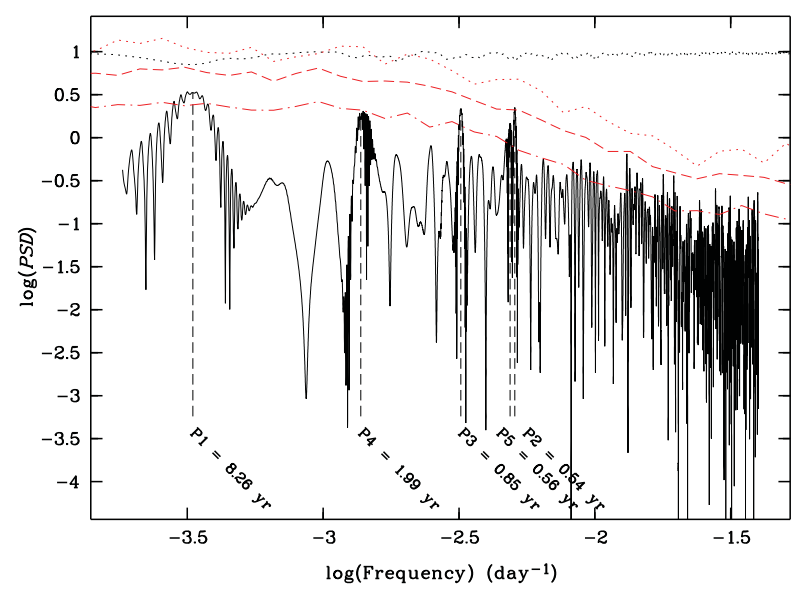

Figure 1. Periodicity analysis results for AO $0235+164$.

glasses. Those filters matched with the standard B, V (Johnson), $R_{C}$ and $I_{C}$ (Cousins) passbands.

Variation: The magnitude determinations is described in our recent paper (Fan et al. 2014). The photometry results indicate the largest variation of 4.88 mag from $R=14.20$ to $R=19.08 \mathrm{mag}$.

Periodicity Analysis: We performed a Power Spectral Analysis (PSA, or Fourier Analysis, or power-density spectrum analysis) to detect possible harmonic components, and gives some quantitative criterion for the detection of a possible periodic signal as we did in our previous papers (Fan et al. 2006; Fan et al. 2014). Our results show periods: $P_{1}=$ 8.26 yr, $P_{2}=0.55 \mathrm{yr}, P_{3}=0.85 \mathrm{yr}, P_{4}=1.99$ yr; see Fig. 1.

\section{Acknowledgements}

The work is partially supported by the National Natural Science Foundation of China (NSFC 10633010, NSFC 11173009, NSFC 11203007, NSFC 11403006), GDUPS(2009) and (10A027S), and supports for Astrophysics Key Subjects of Guangdong Province and Guangzhou City. The Abastumani team acknowledges the financial support of the project FR/639/6-320/12 by the Shota Rustaveli National Science Foundation under contract 31/76. Thanks are given to Profs. J. S. Chen and Y. Y. Zhou for useful discussions.

\section{References}

Chen, Y. J. \& Jiang, D. R., 2001, A\& A, 376, 69

Fan, J. H., Kurtanidze, O., Liu, Y., et al. 2014, ApJS, 213, 26

Fan, J. H., Lin, R. G., Xie, G. Z., et al. 2002, A\& $A$, 381, 1

Fan, J. H., Tao, J., Qian, B. C., Gupta, A. C., \& Liu, Y. 2006, PASJ, 58, 797

Gupta, A. C., Fan, J. H., Bai, J. M., \& Wagner, S. J., 2008, AJ, 135, 1384

Kurtanidze, O. M., Tetradze, S. D., Richter, G. M., et al. 2009, ASPC, 408, 266

Nikolashvili, M. \& Kurtanidze, O. 2007, IAUS, 238, 419

Ostorero, L., Villata, M., \& Raiteri, C. M. 2004, A\&A 419, 913

Peng, B. \& de Bruyn, A. G. 2004, ApJ, 610, 151

Rani, B., Wiita, P. J., \& Gupta, A. C. 2009, ApJ, 696, 2179

Romero, G. E., Cellone, S. A., \& Combi, J. A. 2000, $A \& A$ A, 360, 47

Romero, G. E., Fan, J. H., \& Nuza, S. E. 2003, ChJAA (RAA), 3, 513

Wang, H. T. 2014, ApESSS, 351, 281

Webb, J. R., Smith, A. G., Leacock, R. J. et al. 1998, AJ, 95, 374 